\title{
Pulse electromagnetic fields enhance extracellular electron transfer in magnetic bioelectrochemical systems
}

\author{
Huihui Zhou', Bingfeng Liu', Qisong Wang ${ }^{2}$, Jianmin Sun ${ }^{1,3}$, Guojun Xie ${ }^{1}$, Nanqi Ren ${ }^{1}$, Zhiyong Jason Ren ${ }^{4}$ \\ and Defeng Xing ${ }^{1 *}$ (i)
}

\begin{abstract}
Background: Microbial extracellular electron transfer (EET) is essential in driving the microbial interspecies interaction and redox reactions in bioelectrochemical systems (BESs). Magnetite $\left(\mathrm{Fe}_{3} \mathrm{O}_{4}\right)$ and magnetic fields (MFs) were recently reported to promote microbial EET, but the mechanisms of MFs stimulation of EET and current generation in BESs are not known. This study investigates the behavior of current generation and EET in a state-of-the-art pulse electromagnetic field (PEMF)-assisted magnetic BES (PEMF-MBES), which was equipped with magnetic carbon particle ( $\left.\mathrm{Fe}_{3} \mathrm{O}_{4} @ \mathrm{~N}-\mathrm{mC}\right)$-coated electrodes. Illumina Miseq sequencing of $16 \mathrm{~S}$ rRNA gene amplicons was also conducted to reveal the changes of microbial communities and interactions on the anode in response to magnetic field.

Results: PEMF had significant influences on current generation. When reactors were operated in microbial fuel cell (MFC) mode with pulse electromagnetic field (PEMF-MMFCs), power densities increased by $25.3-36.0 \%$ compared with no PEMF control MFCs (PEMF-OFF-MMFCs). More interestingly, when PEMF was removed, the power density dropped by $25.7 \%$, while when PEMF was reintroduced, the value was restored to the previous level. Illumina sequencing of $16 \mathrm{~S}$ rRNA gene amplicon and principal component analysis (PCA) based on operational taxonomic units (OTUs) indicate that PEMFs led to the shifts in microbial community and changes in species evenness that decreased biofilm microbial diversity. Geobacter spp. were found dominant in all anode biofilms, but the relative abundance in PEMF-MMFCs (86.1-90.0\%) was higher than in PEMF-OFF-MMFCs (82.5-82.7\%), indicating that the magnetic field enriched Geobacter on the anode. The current generation of Geobacter-inoculated microbial electrolysis cells (MECs) presented the same change regularity, the accordingly increase or decrease corresponding with switch of PEMF, which confirmed the reversible stimulation of PEMFs on microbial electron transfer.

Conclusion: The pulse electromagnetic field (PEMF) showed significant influence on state-of-the-art pulse magnetic bioelectrochemical systems (PEMF-MBES) in terms of current generation and microbial ecology. EET was instantaneously and reversibly enhanced in MBESs inoculated with either mixed-culture or Geobacter. PEMF notably decreased bacterial and archaeal diversities of the anode biofilms in MMFCs via changing species evenness rather than species richness, and facilitated specific enrichment of exoelectrogenic bacteria (Geobacter) on the anode surface. This study demonstrates a new magnetic approach for understanding and facilitating microbial electrochemical activities.
\end{abstract}

Keywords: Magnetic bioelectrochemical system (MBES), Microbial fuel cell, Microbial electrolysis cell, Magnetic field, Pulse electromagnetic field, Magnetic carbon particles, Microbial community

\footnotetext{
*Correspondence: dxing@hit.edu.cn

${ }^{1}$ School of Environment, State Key Laboratory of Urban Water Resource

and Environment, Harbin Institute of Technology, 73 Huanghe Road,

Nangang District, P.O. Box 2614, Harbin 150090, Heilongjiang, China

Full list of author information is available at the end of the article
} 


\section{Background}

Bioelectrochemical system (BES) has gained increased attention due to their versatile functions in energy production, environmental remediation, electrosynthesis, and remoting sensing [1-3]. Microbial extracellular electron transfer (EET) is essential in driving the microbial interspecies interaction and redox reactions in both natural and engineered environments. Three types of EET pathways were identified: outer-membrane $c$-type cytochromes, conductive pili, and electron shuttles [46], and recent studies found an additional EET pathway mediated by conductive minerals [7]. For instance, magnetite $\left(\mathrm{Fe}_{3} \mathrm{O}_{4}\right)$ is a conductive mineral that can be used as electron conduits to facilitate EET of $\mathrm{Fe}(\mathrm{III})$-reducing microorganisms. This also compensates for the lack of OmcS to mediate electron transfer between the pili of Geobacter sulfurreducens and electron donors or acceptors $[8,9]$. Furthermore, electrically conductive magnetite nanoparticles are able to facilitate interspecies electron transfer (IET) between Geobacter sulfurreducens and Thiobacillus denitrificans, which in turn are able to establish a cooperative metabolism [10]. Nowadays, magnetite has been used for a wide range of applications due to its superparamagnetic properties, low mass transfer resistance, and selective separation of immobilized biomolecules and bacteria by applying magnetic fields (MFs) $[11,12]$.

Magnetic fields (MFs) provide a cost-effective and convenient approach in changing microbial activity. It has been used in various kinds of MFs-assisted bioreactors [13-15]. Earlier studies demonstrated that MF was able to promote the biocatalytic processes mediated by cytochrome $\mathrm{c}$ and to enhance electrical output of the biofuel cells through the magnetohydrodynamics [13, 16]. Recent studies showed that electricity production by MFCs was improved significantly by a static MF [17, 18]. The removal of chemical oxygen demand (COD) in wastewater treatment increases when MFs are applied on MFCs [19]. MFs enhanced bioelectrochemical activities of anodic biofilms, while there was no increase in secretion of redox mediators [20]. Previous studies have shown that MFs enhanced the performance of MFCs. No investigation on how magnetite and MFs influence microbial community structures in MFCs that affect EET. Compared with the permanent magnet, the electromagnetic devices with adjustable intensity and direction of magnetic fields can provide use flexibility for the largescale BESs. However, the effect of pulse electromagnetic field (PEMF) on BESs has not been investigated.

Electrodes modified with metal oxide, conducting polymer, and nanocomposite have been studied in order to enhance EET and enrichment of exoelectrogenic bacteria [21-26]. However, magnetic material-modified electrodes have not been applied in BESs. Some properties of magnetite $\left(\mathrm{Fe}_{3} \mathrm{O}_{4}\right)$ are sensitive to oxidation, and agglomeration and low conductivity makes it less than ideal. To circumvent this, $\mathrm{Fe}_{3} \mathrm{O}_{4}$ has been used as hybrid material with various other materials that enhances the properties of the magnetic particles. Polyaniline (PANI) is a carbon-nitrogen precursor that is popular within carbon material preparation because they have an aromatic structure that is conductive to the formation of graphitization structure after carbonization, and it was shown to improve the performance of MFCs as the conductive polymer [27-29] by serving as a conductive and protective shell for magnetite in $\mathrm{Fe}_{3} \mathrm{O}_{4}$ /polyaniline hybrid $[12,30,31]$.

In this study, we hypothesized that the magnetic carbon particle-modified electrodes in BESs may facilitate EET and current production under pulsed magnetic fields. To test this hypothesis, we constructed a new magnetic bioelectrochemical system (MBES) with magnetic carbon particle-modified electrodes, and we developed a pulse electromagnetic field (PEMF) to hopefully enhance microbial extracellular electron transfer. Furthermore, we used molecular biology tools to understand the changes of microbial communities and interactions on the anode in response to PEMF operation in the PEMF-MBES reactors.

\section{Methods}

\section{Synthesis of $\mathrm{Fe}_{3} \mathrm{O}_{4} @ \mathrm{~N}-\mathrm{mC}$ composite}

The fabrication of $\mathrm{Fe}_{3} \mathrm{O}_{4} @ \mathrm{~N}$-mC is previously described (Additional file 1: Fig. S1) [31]. $\mathrm{Fe}_{3} \mathrm{O}_{4} @ \mathrm{~N}-\mathrm{mC}$ was the product of the carbonization of magnetic mesoporous polyaniline composite $\left(\mathrm{Fe}_{3} \mathrm{O}_{4} @ \mathrm{mPANI}\right)$, and the $\mathrm{Fe}_{3} \mathrm{O}_{4} @ \mathrm{mPANI}$ was obtained from aniline polymerization on the surface of the PVP-modified $\mathrm{Fe}_{3} \mathrm{O}_{4}$ particles $\left(\mathrm{Fe}_{3} \mathrm{O}_{4}\right.$-PVP). $\mathrm{Fe}_{3} \mathrm{O}_{4}$-PVP was synthesized using the solvothermal method [32]. Ferric chloride hexahydrate, sodium acetate, and PVP were dissolved in ethylene glycol, and then crystallized at $200{ }^{\circ} \mathrm{C}$ for $8 \mathrm{~h}$. Synthesized $\mathrm{Fe}_{3} \mathrm{O}_{4}$-PVP (black magnetic particles) was separated and recovered using a strong magnet. $\mathrm{Fe}_{3} \mathrm{O}_{4}$-PVP was added to the mixture of tergitol(tm) $x h$ (nonionic) (P123) and sodium dodecyl sulfate (SDS) and dissolved in diluted hydrochloric acid $(1 \mathrm{~mol} / \mathrm{L})$. After $30 \mathrm{~min}$, it was ultrasonically dispersed, and the mixture was placed at $4{ }^{\circ} \mathrm{C}$. The mixture was stirred, and aniline $(0.1 \mathrm{~mol} / \mathrm{L})$ and ammonium persulfate $(0.4 \mathrm{~mol} / \mathrm{L})$ were slowly added into the mixture. After that, the mixture was stirred for an additional $6 \mathrm{~h}$ to obtain $\mathrm{Fe}_{3} \mathrm{O}_{4} @$ mPANI. Finally, the $\mathrm{Fe}_{3} \mathrm{O}_{4} @ \mathrm{mPANI}$ was carbonized with nitrogen at a heating rate of $3{ }^{\circ} \mathrm{C} \mathrm{min}^{-1}$ to $700{ }^{\circ} \mathrm{C}$ and kept at $700{ }^{\circ} \mathrm{C}$ for $6 \mathrm{~h}$ in a tube furnace to synthesize $\mathrm{Fe}_{3} \mathrm{O}_{4} @ \mathrm{~N}-\mathrm{mC}$. 


\section{Configuration and operation of magnetic BESs}

Single-chamber microbial fuel cells (MFCs) (cylindrical chamber, $28 \mathrm{~mL}$ ) were constructed, as previously described [33]. The anode was stainless steel mesh (SSM, type SUS304) coated with $\mathrm{Fe}_{3} \mathrm{O}_{4} @ \mathrm{~N}-\mathrm{mC}\left(5 \mathrm{mg} / \mathrm{cm}^{2}\right)$. The cathode rolled the activated carbon-PTFE air cathode [34]. The effective area of each cathode and anode was $7 \mathrm{~cm}^{2}$, respectively, which were connected by titanium wires through an external resistance of $1000 \Omega$. The wires on the electromagnetic launcher were intertwined on the external walls of the anode chamber in order to obtain a pulse electromagnetic field (symmetric periodic square wave) with the frequency of $100 \mathrm{~Hz}$. A magnetic intensity of $5 \mathrm{uT}$ was applied on the reactors as they were $5 \mathrm{~cm}$ away from electric magnetic field analyzer (EHP-200A, Narda, Inc., Italy). Activated sludge from a wastewater treatment plant (Harbin, China) was used to inoculate the magnetic MFCs (MMFCs). MMFCs were operated in both the presence (PEMF-MMFCs) and absence of PEMFs (PEMF-OFF-MMFCs). The cultured solution contained (per liter) $2 \mathrm{~g}$ sodium acetate, $50 \mathrm{mM}$ phosphate buffer solution (PBS) $\left(11.55 \mathrm{~g} / \mathrm{L} \mathrm{Na}_{2} \mathrm{HPO}_{4} \cdot 12 \mathrm{H}_{2} \mathrm{O}\right.$, $2.77 \mathrm{~g} / \mathrm{L} \mathrm{NaH} \mathrm{PO}_{4} \cdot 2 \mathrm{H}_{2} \mathrm{O}, 0.31 \mathrm{~g} / \mathrm{L} \mathrm{NH}_{4} \mathrm{Cl}$ and $0.13 \mathrm{~g} / \mathrm{L}$ $\mathrm{KCl}$ ), $10 \mathrm{~mL}$ mineral solution, and $10 \mathrm{~mL}$ vitamin solution [35]. For each test, there was a duplicate MMFC in operation in the fed-batch mode at a constant temperature $\left(25 \pm 2{ }^{\circ} \mathrm{C}\right)$.

For the pure culture test, single-chamber microbial electrolysis cells (MECs) were constructed using a 250$\mathrm{mL}$ anaerobic bottle with a liquid volume of $100 \mathrm{~mL}$. The anode was carbon paper and the cathode rolled activated carbon-PTFE. The effective area of each cathode and anode was $5.4 \mathrm{~cm}^{2}(1.8 \mathrm{~cm} \times 3 \mathrm{~cm})$, respectively. A fixed voltage of $0.6 \mathrm{~V}$ was applied to MECs by a programmable power source (3645A, Array, Inc.). All reactors were inoculated with Geobacter sulfurreducens PCA (ATCC 51573) purchased from American Type Culture Collection (ATCC). MECs were filled with the medium containing (per liter, $\mathrm{pH}$ 6.8): $0.82 \mathrm{~g}$ sodium acetate, $0.1 \mathrm{~g} \mathrm{KCl}$, $1.5 \mathrm{~g} \mathrm{NH}_{4} \mathrm{Cl}, 0.6 \mathrm{~g} \mathrm{NaH}_{2} \mathrm{PO}_{4}, 2 \mathrm{~g} \mathrm{NaHCO}_{3}, 10 \mathrm{~mL}$ mineral solution, and $10 \mathrm{~mL}$ vitamin solution. Prior to use, the medium was flushed with $\mathrm{N}_{2}-\mathrm{CO}_{2}$ (80:20). All MECs were operated at a constant temperature $\left(35 \pm 2{ }^{\circ} \mathrm{C}\right)$. The pulse electromagnetic fields were applied on MECs by a sequential "ON" and "OFF" process to analyze the instantaneous effect on the current generation by MECs. The current in the circuit was conducted by measuring the voltage across a high-precision resistor $(10 \Omega)$.

\section{Analytical and electrochemical techniques}

MMFC voltage over the external resistance was automatically recorded every 5 min by a multi-channel data acquisition system (Model 2700 with 7702 module,
Keithley Instruments Inc., USA). Both linear sweep voltammetry (LSV) and electrochemical impedance spectroscopy (EIS) were measured on the two-electrode mode. This analyzed the entire cell with the anode as the working electrode and the cathode as the reference electrode by using Autolab Potentiostat/Galvanostat (Autolab PGSTAT 128 N, MetrohmAutolab Inc., Netherlands). Polarization and power density curves were plotted based on linear sweep voltammetry (LSV), which was conducted from open-circuit voltage (OCV) to $0 \mathrm{~V}$ with a scan rate of $0.1 \mathrm{mV} / \mathrm{s}$. Electrochemical impedance spectroscopy (EIS) was conducted with a frequency range of $100 \mathrm{kHz}-10 \mathrm{mHz}$ at the OCV of MFCs. The Nyquist plots were analyzed using NOVA software. The impedance spectra were analyzed by fitting to the equivalent circuit (EC): $R(\mathrm{Q}[\mathrm{RW}])$. The EC contained $R_{\mathrm{s}}, R_{\mathrm{p}}, W$, and CPE four parts. $R_{\mathrm{s}}$ represents ohmic resistance; $R_{\mathrm{p}}$ represents activation resistance; constant phase element (CPE) represents the electrical double-layer capacitor; and Warburg (W) represents diffusion resistance [36].

X-ray diffraction (XRD) characterization was obtained on a Bruker AXS D8-Advanced diffractometer with $\mathrm{CuK \alpha}$ radiation $(\lambda=1.5418 \AA)$. Raman experiments were performed on a LabRAM XploRA Raman microscope using a $532 \mathrm{~nm}$ excitation line from an argon-ion laser with a power of $0.15 \mathrm{~mW}$. The magnetization curve was carried out on Quantum Design MPMS-7 SQUID magnetometer at $300 \mathrm{~K}$ under varying magnetic fields. The samples were analyzed by Fourier transform infrared spectroscopy (FTIR) on Perkin Elmer 100 spectrometer from 400 to $4000 \mathrm{~cm}^{-1}$ and X-ray photoelectron spectroscopy (XPS; PH1-5700 ESCA system, US) using a hemispherical analyzer and an aluminum anode (monochromatic $\mathrm{Al} \mathrm{Ka}$, $1486.6 \mathrm{eV}$ ) as the source (at $12-14 \mathrm{kV}$ and $10-20 \mathrm{~mA}$ ). The microstructures of samples were observed by scanning electron microscope (SEM; Helios Nanolab600i, FEI, USA) and transmission electron microscope (TEM; JEM2100 electron microscope, JEOL, Japan) [37].

\section{Illumina sequencing of $16 \mathrm{~S}$ rRNA gene amplicons}

After 2 months of operation, the anodes of MMFCs were cut and fragmented using sterile scissors [38]. Genomic DNA of the anode biofilms were extracted using the PowerSoil DNA Isolation Kit (Mo Bio Laboratories, Inc., Carlsbad, CA) according to the manufacturer's instructions. DNA from two pieces of an electrode were isolated and mixed equally for PCR amplification after DNA concentration was determined using Qubit fluorometer (Thermo Fisher Scientific). V4-V5 regions of bacterial and archaeal 16S rRNA genes were amplified using universal primers: 515F (5'-GTGCCAGCMGCCGCGGTAA-3') and 907R (5'-CCGTCAATTCCTTTGAGTTT-3'); U519F (5'-CAGYMGCCRCGGKAAHACC-3') and 806R 
(5'-GGACTACNSGGGTMTCTAAT-3'). PCR products were examined using agarose gel electrophoresis and then purified using a PCR purification kit (Qiagen, Germany). High-throughput sequencing of $16 \mathrm{~S}$ rRNA gene amplicons was carried out on the Illumina HiSeq 2500 platform.

The sequencing reads were initially assembled into raw tags. Effective reads were obtained after the raw reads were trimmed, filtered, and removed chimeric sequences using QIIME software (http://qiime.org). Operational taxonomic units (OTUs) were determined at a 97\% sequence similarity cutoff using UPARSE software [39]. The representative OTUs were selected according to the highest frequency and assigned to a taxonomic identification by using the RDP Classifier at a 0.8 confidence threshold [40]. Species diversities (Observed-species,
Chao1, Shannon, Simpson, and ACE) were calculated by QIIME software.

\section{Results}

Synthesis and characterization of $\mathrm{Fe}_{3} \mathrm{O}_{4} @ \mathrm{~N}$-mC modified electrodes

A well-defined core-shell structure of $\mathrm{Fe}_{3} \mathrm{O}_{4} @ \mathrm{~N}$-mC composite was synthesized. As shown in TEM micrographs, a dark inner core $\left(\mathrm{Fe}_{3} \mathrm{O}_{4}\right)$ was coated with a lighter carbon skin, and SEM images showed that the shell contained by many tiny nanoparticles around $30 \mathrm{~nm}$ in length (Additional file 1: Fig. S2). The Raman spectrum of $\mathrm{Fe}_{3} \mathrm{O}_{4} @ \mathrm{~N}-\mathrm{mC}$ showed two peaks at around 1330 and $1600 \mathrm{~cm}^{-1}$ that could be labeled as the D-band and G-band of disordered and graphitized carbons (Fig. 1a) [41], demonstrating that the carbon structure existed on the surface of $\mathrm{Fe}_{3} \mathrm{O}_{4}$.
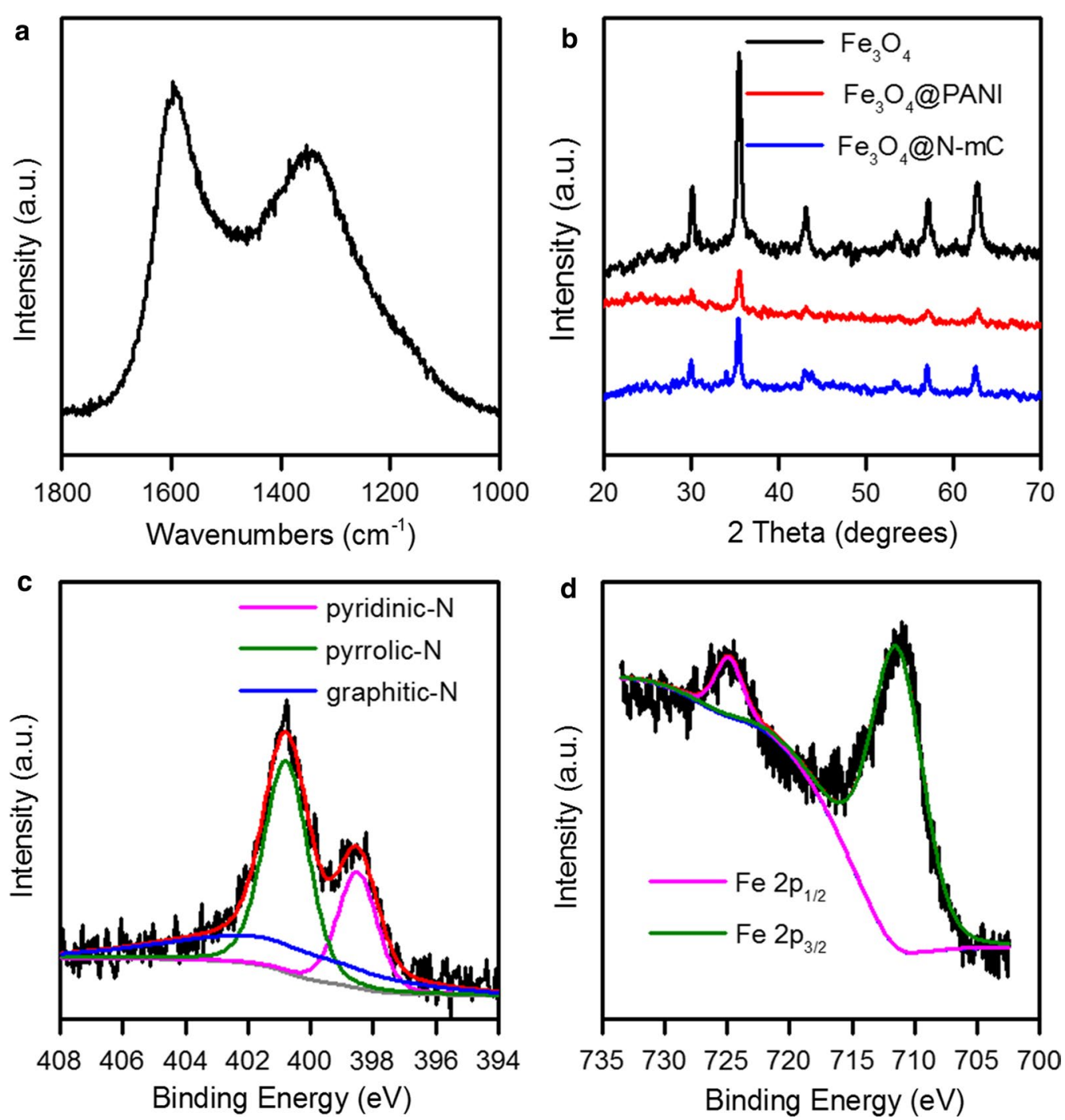

Fig. 1 Characterizations of synthesized $\mathrm{Fe}_{3} \mathrm{O}_{4} @ \mathrm{~N}-\mathrm{mC}$ using Raman spectra (a), XRD (b), and high-resolution XPS [N 1s (c) and Fe 2p (d)] 
The XPS survey spectrum of $\mathrm{Fe}_{3} \mathrm{O}_{4} @ \mathrm{~N}-\mathrm{mC}$ confirmed the existences of $\mathrm{C}, \mathrm{O}, \mathrm{N}$, and Fe elements; the element contents were also measured (Additional file 1: Fig. S3). The high-resolution $\mathrm{N}$ 1s scan revealed three typical $\mathrm{N}$ species, namely pyridinic $\mathrm{N}(398.5 \mathrm{eV})$, pyrrolic $\mathrm{N}$ (400.4), and graphitic N (401.3) (Fig. 1c) [42]. The FTIR characteristic peaked at $1590 \mathrm{~cm}^{-1}$ thus belonging to the $-\mathrm{NH}_{2}$ deformation vibration. This also indicated the successful $\mathrm{N}$-functionalization on the $\mathrm{Fe}_{3} \mathrm{O}_{4}$ surface [43]. Peaks at 1605, 1494, 1296, 1235, and 1148, $830 \mathrm{~cm}^{-1}$ were in good agreement with PANI [44], which disappeared in $\mathrm{Fe}_{3} \mathrm{O}_{4} @ \mathrm{~N}-\mathrm{mC}$ due to the carbonization process. Additionally, two peaks at 470 and $585 \mathrm{~cm}^{-1}$ were categorized as the $\mathrm{Fe}-\mathrm{O}$ stretching vibrations of $\mathrm{Fe}_{3} \mathrm{O}_{4}$ (Additional file 1: Fig. S4) [45]. The XRD patterns of $\mathrm{Fe}_{3} \mathrm{O}_{4}, \mathrm{Fe}_{3} \mathrm{O}_{4} @ \mathrm{PANI}$, and $\mathrm{Fe}_{3} \mathrm{O}_{4} @ \mathrm{~N}$-mC showed five significant diffraction peaks that were in good agreement with cubic lattice of $\mathrm{Fe}_{3} \mathrm{O}_{4}$ (JCPDS No. 19-0629) (Fig. 1b). When $\mathrm{Fe}_{3} \mathrm{O}_{4}$ was coated with a layer of PANI, the intensity of its diffraction peaks decreased, and the intensity of the peaks increased after the carbonization. The standard S-shaped hysteresis curve of the $\mathrm{Fe}_{3} \mathrm{O}_{4} @ \mathrm{~N}-\mathrm{mC}$ depict its magnetic behavior (Additional file 1: Fig. S5). Fe 2p high-resolution spectrum indicated that Fe species were in the form of Fe $2 \mathrm{p}_{1 / 2}(725 \mathrm{eV})$ and $\mathrm{Fe} 2 \mathrm{p}_{3 / 2}(711.3 \mathrm{eV})$ (Fig. 1d) [46]. These results are proof that the $\mathrm{Fe}_{3} \mathrm{O}_{4} @ \mathrm{~N}-\mathrm{mC}$ composite was successfully synthesized.

\section{PEMF enhanced current generation by MMFCs}

The performance of magnetic MFCs (MMFCs) equipped with $\mathrm{Fe}_{3} \mathrm{O}_{4} @ \mathrm{~N}$-mC-coated anodes was characterized in the presence or absence of PEMF. MMFCs exposed to PEMF (PEMF-MMFCs) started up faster as its voltage increased rapidly at $60 \mathrm{~h}$ compared with the MFCs without PEMF (PEMF-OFF-MMFCs) (95 h), indicating that MF accelerated the start-up of MMFCs (Fig. 2). The peak voltage of PEMF-MMFC (508 mV,1000 $\Omega$ of external resistance) was slightly higher than that of PEMF-OFF-MMFC $(485 \mathrm{mV})$ during the initial start-up stage. MFCs had a similar peak voltage $(503 \mathrm{mV})$ during the stable operation stage (12-50 days). The peak voltage of PEMF-MMFC increased to $529 \mathrm{mV}$ after the long-term operation, versus $483 \mathrm{mV}$ of PEMF-OFFMMFC. PEMF-MMFCs demonstrated a longer discharge period of batch cycle $(\sim 118 \mathrm{~h})$ than PEMF-OFF-MMFCs $(\sim 78 \mathrm{~h})$ and higher peak voltage. This suggests that exoelectrogenic biofilms in PEMF-MMFCs had a higher capability of extracellular electron transfer than those in PEMF-OFF-MMFCs.

In the medium and high current density regions of polarization curves, PEMF-MMFC showed higher potential than PEMF-OFF-MMFC when current densities

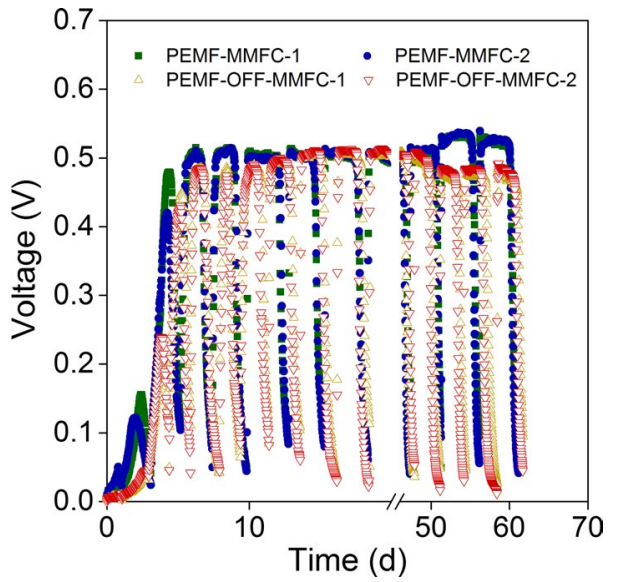

Fig. 2 Voltage production of MMFCs in the presence (PEMF-MMFCs) and absence of PEMF (PEMF-OFF-MMFCs) at external resistance of $1000 \Omega$. Numbers represent duplicate MMFC reactors

were the same, suggesting a lower activation resistance and a lower diffusion resistance of PEMF-MMFC (Fig. 3a). PEMF-MMFC obtained the highest maximum power densities of $0.94-1.02 \mathrm{~W} / \mathrm{m}^{2}$, which is $25.3-36.0 \%$ higher than the $0.74-0.75 \mathrm{~W} / \mathrm{m}^{2}$ obtained by PEMFOFF-MMFC (Fig. 3b). The maximum power density of PEMF-MMFC decreased 25.7\% when PEMF was turned off, which was then restored perfectly when PEMF was reintroduced (Fig. 3c).

On the basis of the Ohm's law, the internal resistances of MMFCs were calculated in Additional file 1: Table S1. PEMF-MMFCs showed lower internal resistance of 61.4-69.6 $\Omega$ when compared to PEMF-OFF-MMFCs (87.2-88.0 $\Omega$ ), indicating the addition of PEMF resulted in the decrease of the internal resistance of MMFCs. According to electrochemical impedance spectroscopy (EIS) analysis, PEMF-MMFCs had similar ohmic resistance as PEMF-OFF-MMFCs, but its activation resistance ranged from 2.83 to $8.65 \Omega$, much lower than the range of 17.5-17.9 $\Omega$ measured for PEMF-OFF-MMFCs (Fig. 4). PEMF-MMFC had lower diffusion resistance since its conductivity of 138-143 mho was higher than 119126 mho of PEMF-OFF-MMFC. Thus, PEMF decreases the internal resistance of MMFCs by reducing its activation resistance and diffusion resistance.

\section{PEMF and magnetic anode influenced bacterial and archaeal community structures}

Reads were obtained from each sample after filtering and removing chimera (Additional file 1: Table S2), ranging from 37,730 to 59,939 with a length of 373 . The Good's coverage estimator of $0.999-1$ indicated that almost the entire microbial community for each sample was tracked using Illumina sequencing of $16 \mathrm{~S}$ rRNA gene amplicons. 

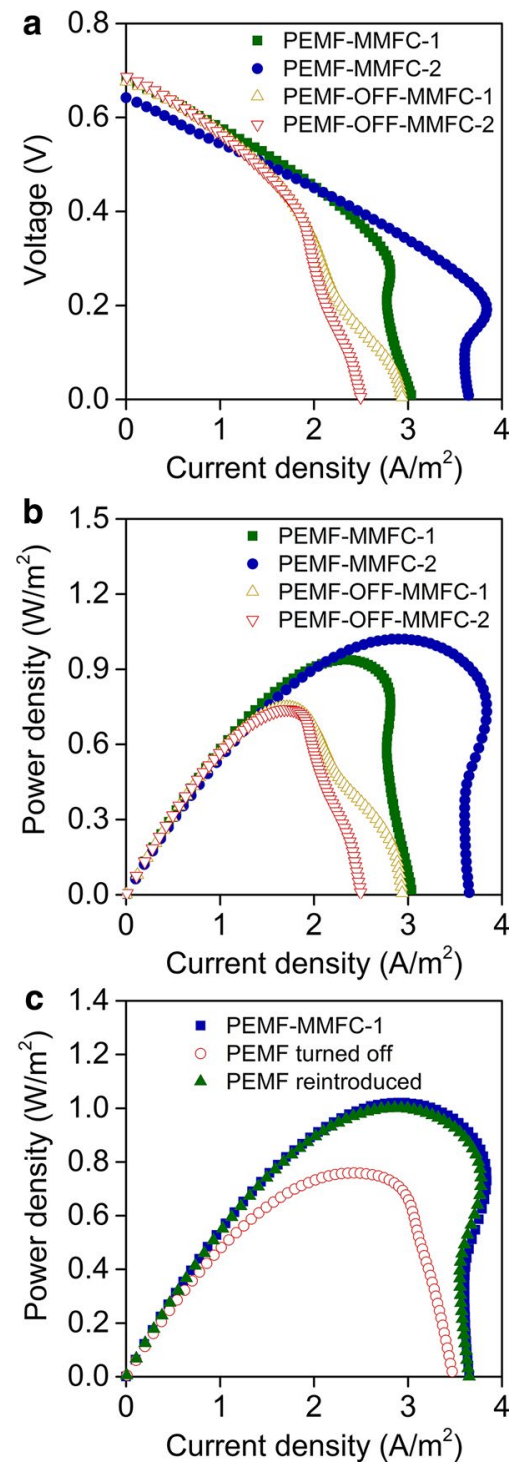

Fig. 3 Polarization $(\mathbf{a})$ and power density $(\mathbf{b}, \mathbf{c})$ curves of MMFCs under the on and off cycle of PEMF

Total operational taxonomic units (OTUs) of 146 and 141 in bacteria, along with 95 and 114 in archaea were detected for the anode biofilms of PEMF-MMFCs at a threshold of 97\%, with OTUs of 149 and 164 in bacteria, and 115 and 128 in archaea for the anode biofilms of PEMF-OFF-MMFCs. The observed OTUs and expected richness (Chao1 and ACE estimators) were similar between both the PEMF-OFF-MMFC-1 and the PEMF-MMFC-1; however, the Shannon's and the Simpson's diversity indices of PEMF-MMFCs were lower than that of PEMF-OFF-MMFCs. This suggests that the application of PEMF on MMFCs resulted in the decrease in species evenness, rather than species richness. Principal
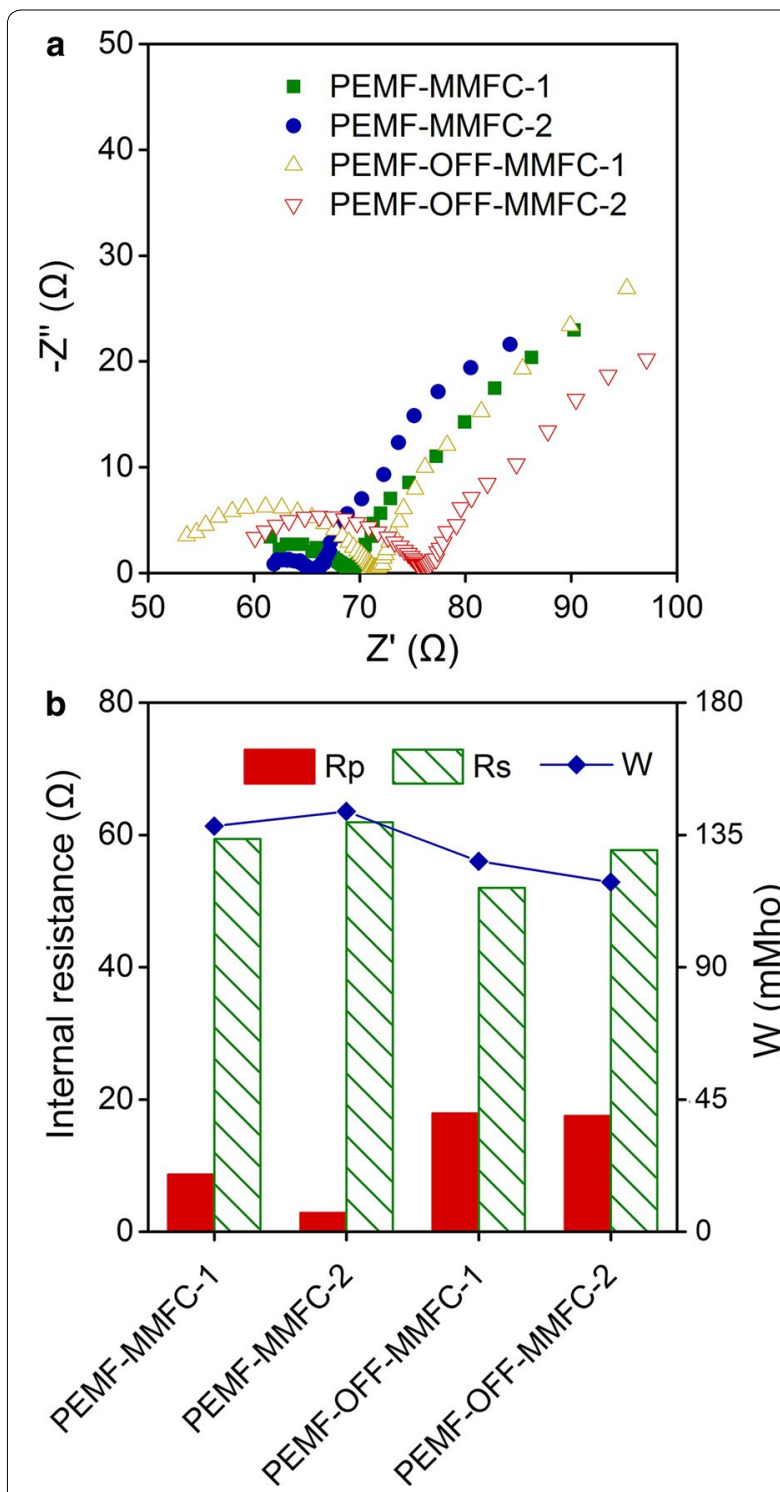

Fig. 4 Nyquist plots (a) and internal resistances of PEMF-MMFCs and PEMF-OFF-MMFCs analyzed by fitting to the equivalent circuit: $R$ ( $Q$ $[\mathrm{RW}])(\mathbf{b})$

component analysis (PCA) showed that there were distinct separations of bacterial and archaeal communities between PEMF-MMFCs and PEMF-OFF-MMFCs, indicating that PEMF led to significant shifts in the microbial community structures (Fig. 5).

\section{PEMF and magnetic anode facilitated the enrichment of exoelectrogenic bacteria}

The predominant phyla present were Proteobacteria and Bacteroidetes in all obtained samples (Fig. 6a). The relative amount of Proteobacteria in PEMF-MMFCs (86.7 and 90.5\%) was slightly higher than that in 


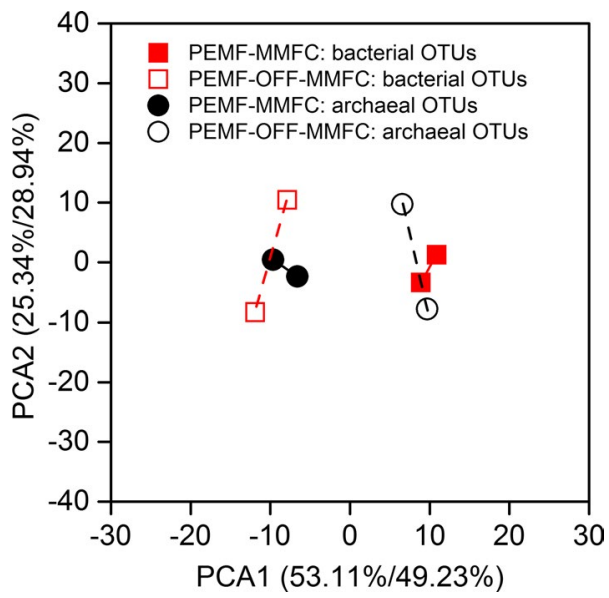

Fig. 5 Principle component analysis (PCA) based on bacterial and archaeal operational taxonomic units of the anode biofilms of PEMFMMFCs and PEMF-OFF-MMFCs

PEMF-OFF-MMFCs (84.2 and 84.7\%). However, the relative abundance of Bacteroidetes in PEMF-MMFCs (6.9 and 9.5\%) was lower than that in PEMF-OFF-MMFCs (10.3 and 11.7\%). At class level, the relative abundance of Deltaproteobacteria (86.4 and 90.2\%) in PEMF-MMFCs was higher than that in PEMF-OFF-MMFCs (83.1 and 83.2\%) (Fig. 6b). The majority of predominant populations were affiliated with Geobacter in MMFCs (Fig. 7). The relative abundance of Geobacter in PEMF-MMFCs (86.1 and $90.0 \%$ ) was higher than that in PEMF-OFFMMFCs (82.5 and 82.7\%), implying that PEMF enabled enrichment of exoelectrogens on the anode. Archaeal communities in MMFCs were dominated by Cenarchaeum symbiosum, accounting for $99.5-99.8 \%$ of the archaea, indicating that PEMF did not predominantly shift archaeal populations, although it did shift the species diversity of archaeal communities.

\section{PEMF reversibly stimulated current generation by Geobacter}

Since the predominant population in all anode biofilms was affiliated with Geobacter sulfurreducens (similarity of 99-100\%), the effects of PEMF on current generation by G. sulfurreducens in microbial electrolysis cells (MECs) were evaluated. Current generation by Geobacter in PEMF-MMEC was higher than that in PEMF-OFF-MEC (Fig. 8a). In order to evaluate instantaneous response of Geobacter to PEMF during steady operation stage, PEMF was periodically turned off (PEMF-OFF-MEC) and on (PEMF-MEC) at 8-h interval. Results show that current generation by Geobacter corresponded very well with the PEMF cycle, during which current increased with the application of PEMF and decreased with the absence of
PEMF. Interestingly, PEMF effectively stimulated current generation by Geobacter in MECs, no matter the initial MECs were operated in the presence or absence of PEMF (Fig. 8b).

\section{Discussion \\ Pulse electromagnetic field stimulated magnetic BES (PEMF-MBES)}

This study reports for the first time that pulse electromagnetic field (PEMF) stimulated current generation in magnetic BES (MBES) with $\mathrm{Fe}_{3} \mathrm{O}_{4} @ \mathrm{~N}$-mC-coated electrode (PEMF-MBES). Magnetic MFCs (MMFCs) that were exposed to PEMF presented fast biofilm acclimation, and the maximum power density increased by $25.3-$ $36.0 \%$ compared with MMFCs without PEMF (Fig. 3b). Such stimulation was further confirmed by reversible effects of PEMF, where power output was decreased by 25.7\% when PEMF was off and resumed to previous level when it was backed on. This phenomenon where PEMF prolonged the discharge period of batch cycle and enhanced the voltage output was consistent with the previous study on the effect of permanent magnetic field on MFCs [20]. Polarization curves showed that PEMF was unable to enhance the potential of open-circuit MFCs (Fig. 3a), implying that PEMF impacted the activity of the anode biofilms. The electrochemical impedance spectroscopy (EIS) analyses showed that when PEMF was present there was a decrease in the activation losses and mass transfer losses (Fig. 3b), which facilitated system mass transfer and consistent with previous studies [18]. Reversible effects of PEMF on current generation by BES provided an instant and controllable method to stimulate EET, which is important for revealing the mechanism of EET.

Recent studies showed that centimeter-long electron transport in marine sediments via conductive minerals is possible [47]. This suggests that the planktonic microbes in the solution of MECs can be trapped and immobilized by magnetic carbon nanoparticles $\left(\mathrm{Fe}_{3} \mathrm{O}_{4} @ \mathrm{~N}-\mathrm{mC}\right)$ and deposited around the anode by controlling magnetic field based on the enhancement capacity requirements of EET. Magnetic $\mathrm{Fe}_{3} \mathrm{O}_{4} @ \mathrm{~N}-\mathrm{mC}$ may also be used as the cathode catalyst. Thus, to improve PEMF-MBES, the effects of magnetic intensity and pulse repetition frequency of PEMF and magnetic carbon nanoparticles on extracellular electron transfer of exoelectrogens need to be investigated in future studies.

\section{PEMF led to shifts in bacterial community diversity in MMFCs}

Although several studies have reported the effects of permanent magnetic field on electricity generation in MFCs, this study investigates the response of microbial 

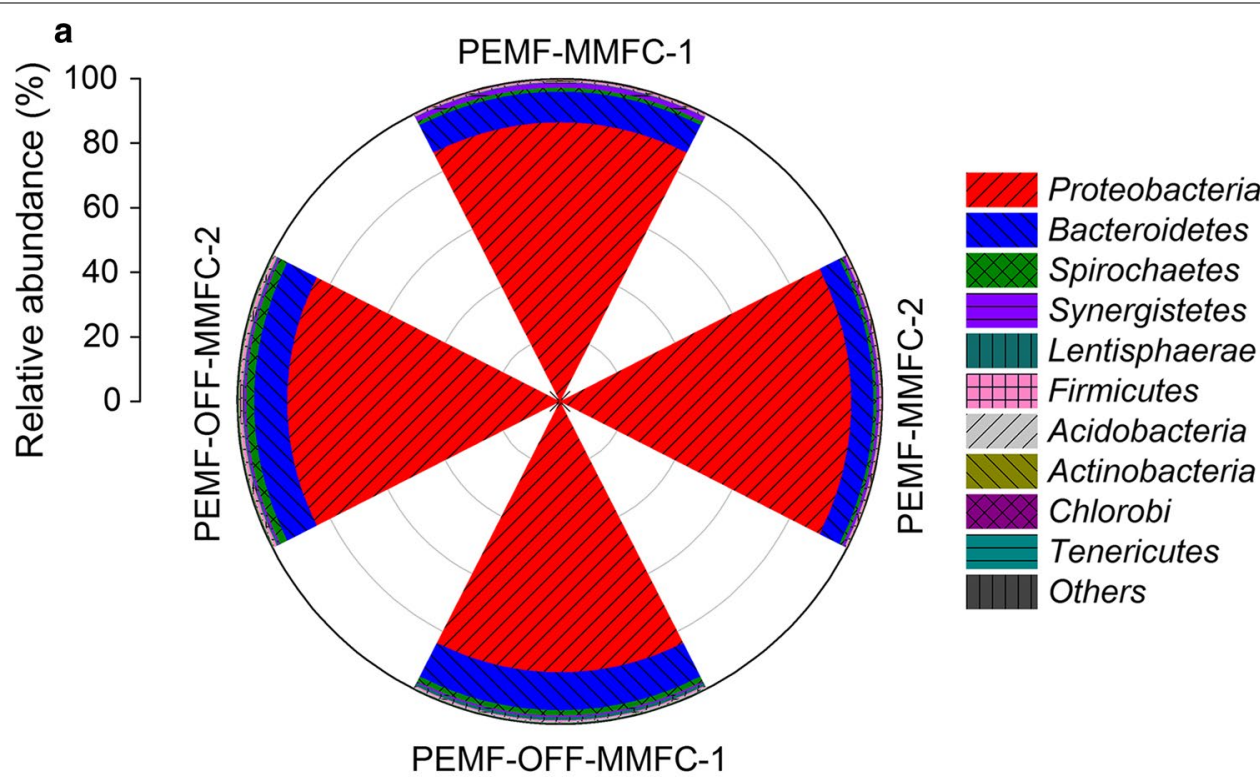

b
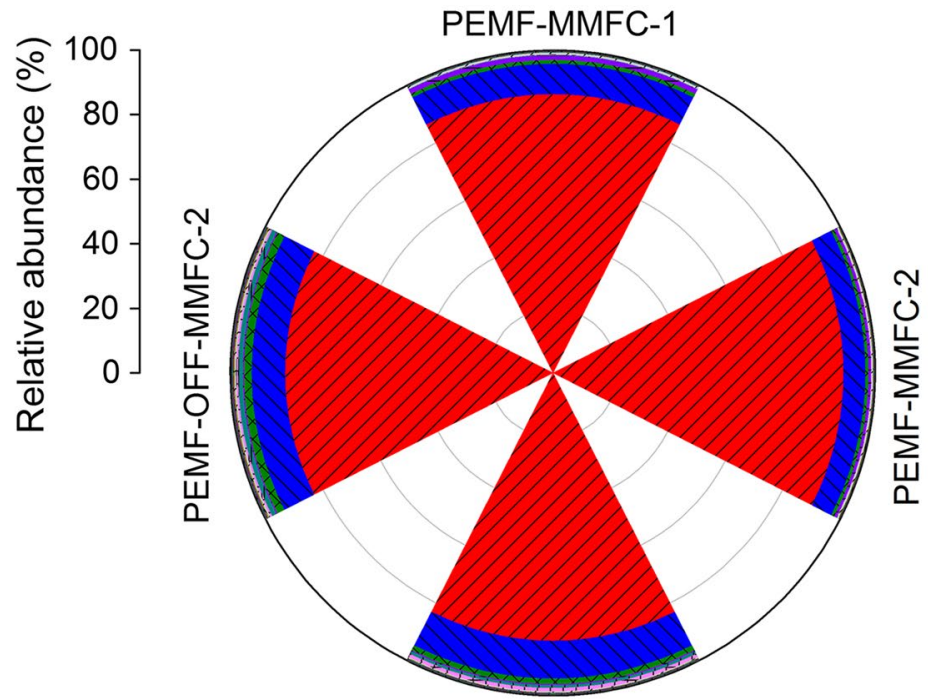

VI/taproteobacteria

Bacteroidia

Spirochaetes

Synergistia

Betaproteobacteria

\# Lentisphaeria

1//, Clostridia

11 Holophagae

Actinobacteria

Erysipelotrichi

Others

PEMF-OFF-MMFC-1

Fig. 6 Taxonomic classification of bacterial 16S rRNA sequences from bacterial communities of PEMF-MMFCs and PEMF-OFF-MMFCs at the phylum level (a) and class level (b). The relative abundances of the top 10 phyla and classes are shown

community to magnetic fields and elucidates the ecological response that resulted in the improved performance. The diversity indices show an obvious decrease in bacterial and archaeal diversity of the anode biofilms when PEMF was present, although it seems that it decreased species evenness rather than species richness.

The relative abundance of Geobacter in MMFCs with $\mathrm{Fe}_{3} \mathrm{O}_{4} @ \mathrm{~N}$-mC went up to $82.5-90.0 \%$ (Fig. 7), implying that $\mathrm{Fe}_{3} \mathrm{O}_{4} @ \mathrm{~N}-\mathrm{mC}$ facilitated specific enrichment of Geobacter on the anode. Previous studies showed that magnetite supplied to rice paddy field soil promoted a specific enrichment of Geobacter species and as well as current generation [8]. A recent study also reported that magnetic particle facilitated enrichment of exoelectrogenic bacteria [48]. The relative abundance of Geobacter in PEMF-MMFCs was $6.6 \%$ higher than that in PEMF-OFF-MMFCs, suggesting that PEMF enhanced the enrichment of Geobacter and therefore produced higher power. Therefore, PEMF and $\mathrm{Fe}_{3} \mathrm{O}_{4} @ \mathrm{~N}-\mathrm{mC}$ coatings are able to shape microbial community structures of the anode biofilms by enriching exoelectrogenic bacteria. 


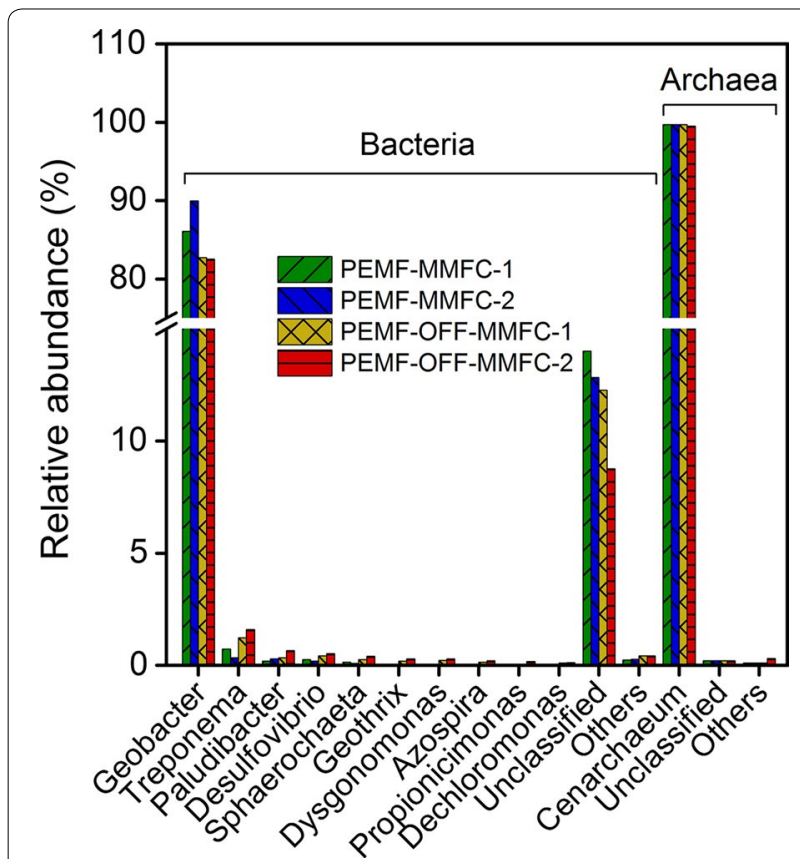

Fig. 7 The relative abundance of predominant bacterial and archaeal populations in the anode biofilms of PEMF-MMFCs and PEMF-OFFMMFCs. The relative abundances of the top 10 genera are shown

Cenarchaeum symbiosum, an uncultivated archaeon, was abundant in the anode biofilms (99.5-99.8\% of relative abundance), which is the sole archaeal symbiont of the marine sponge Axinella Mexicana [49]. Investigating its presumable capacity of extracellular electron transfer of Cenarchaeum symbiosum and its role in the anode biofilms would be significant in understanding exoelectrogenic microorganisms.

\section{The mechanisms of PEMF stimulation for EET in MBES}

This is the first evidence that Geobacter sulfurreducens PCA is able to respond immediately to PEMF (Fig. 8). Another well-known exoelectrogen, Shewanella oneidensis MR-1, showed a similar increase in current generation in MFCs exposed to permanent MF but was unable to significantly enhance mediator secretion [17]. Both pure- and mixed-culture tests have indicated that the effect of PEMF on current generation is instantaneous and reversible; we speculate that PEMF directly enhance capacity of extracellular electron transfer due to the changes in the electroactive activities of cytochrome $c$ and redox enzyme through the magnetohydrodynamic effect, but further evidence is needed in follow-up studies. Electromagnetohydrodynamic effect might reduce the activation resistance and diffusion resistance by affecting the electron motion according to the Lorentz force law. PEMF may also indirectly promote electron
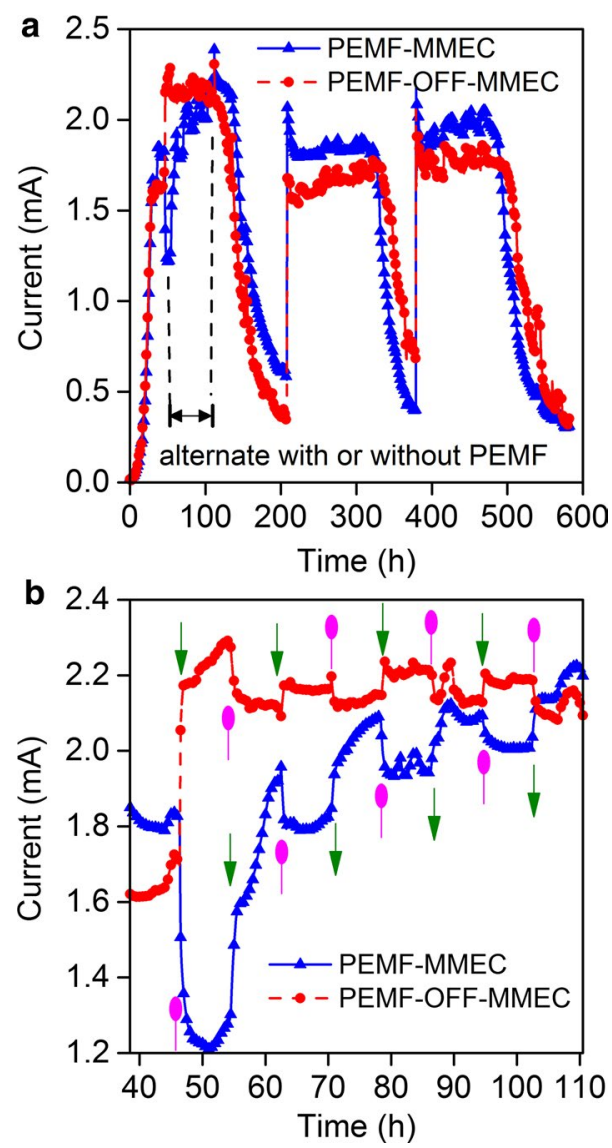

Fig. 8 Current generation by Geobacter sulfurreducens PCA in MECS under the on and off cycle of PEMF. $\mathbf{b}$ presents expanding curves of current generation in MECs during switching of PEMF in an operation cycle (between dish lines in $\mathbf{a}$ ). The green triangle and pink ellipse arrows represent turn on and off of PEMF (b)

transfer by assembling dispersed conductive minerals around exoelectrogenic bacteria. Recent studies reveal that the manganic $\mathrm{Fe}_{3} \mathrm{O}_{4}$ effectively promotes extracellular electron transfer in a manner similar to c-type cytochromes $[9,10]$. To gain insight into the mechanisms of the PEMF stimulation for extracellular electron transfer, the relationships among EET, magnetohydrodynamic effect, pulse frequency and expression and activity of cytochrome and redox enzyme of the exoelectrogenic biofilms need further investigation.

\section{Conclusions}

The pulse electromagnetic field (PEMF) significantly influenced current generation and community deversity of magnetic bioelectrochemical system (PEMF-MBES). The PEMF stimulated current generation when it was applied to MMFCs with magnetic mesoporous carbon particle-coated anode. MBESs inoculated with either mixed-culture or Geobacter showed similar responses 
under PEMF, in which extracellular electron transfer was instantaneously and reversibly enhanced. Illumina sequencing of $16 \mathrm{~S}$ rRNA gene amplicons demonstrated that PEMF notably decreased bacterial and archaeal diversities of the anode biofilms in MMFCs via changing species evenness rather than species richness. The relative abundance of Geobacter was higher in the presence of PEMF, indicating that PEMF-MMFC facilitated specific enrichment of Geobacter on the anode surface.

\section{Additional file}

Additional file 1: Table S1. Comparison of performance of PEMFMMFCS and PEMF-OFF-MMFCs. Table S2. Similarity-based OTUs and species richness and diversity indices at a threshold of $97 \%$. Figure $\mathbf{S 1 .}$ Schematic illustration of the synthesis of $\mathrm{Fe}_{3} \mathrm{O}_{4} @ \mathrm{~N}-\mathrm{mC}$ composite. Figure S2. TEM (a) and SEM (b) images of synthesized $\mathrm{Fe}_{3} \mathrm{O}_{4} @ \mathrm{~N}-\mathrm{mC}$ composite. Figure S3. Survey XPS spectra of synthesized $\mathrm{Fe}_{3} \mathrm{O}_{4} @ \mathrm{~N}-\mathrm{mC}$ composite. The insert represents element contents. Figure S4. FTIR spectra of $\mathrm{Fe}_{3} \mathrm{O}_{4}$, $\mathrm{Fe}_{3} \mathrm{O}_{4} @ m P A N I$ and $\mathrm{Fe}_{3} \mathrm{O}_{4} @ \mathrm{~N}-\mathrm{mC}$ composite. Figure S5. Magnetic hysteresis loops of $\mathrm{Fe}_{3} \mathrm{O}_{4}$ and of synthesized $\mathrm{Fe}_{3} \mathrm{O}_{4} @ \mathrm{~N}$-mC.

\section{Abbreviations}

MFs: magnetic fields; EET: extracellular electron transfer; BESs: bioelectrochemical systems; PEMF: pulse electromagnetic field; PEMF-MBES: pulse electromagnetic field-assisted magnetic $\mathrm{BES} ; \mathrm{Fe}_{3} \mathrm{O}_{4}-\mathrm{PVP}$ : PVP-modified $\mathrm{Fe}_{3} \mathrm{O}_{4}$ particles; $\mathrm{Fe}_{3} \mathrm{O}_{4} @ m P A N I$ : PANI-modified $\mathrm{Fe}_{3} \mathrm{O}_{4}$ particles; $\mathrm{Fe}_{3} \mathrm{O}_{4} @ \mathrm{~N}$-mC: magnetic nitrogen-doped carbon particle; MFC: microbial fuel cell; PEMF-MMFCs: pulse electromagnetic field-assisted magnetic microbial fuel cell; PEMF-OFF-MMFCs: no pulse elctromagnetic field control magnetic microbial fuel cell; PCA: principal component analysis; OTUs: operational taxonomic units; MECs: microbial electrolysis cells; IET: interspecies electron transfer; COD: chemical oxygen demand; PANI: polyaniline; LSV: linear sweep voltammetry; EIS: electrochemical impedance spectroscopy; OCV: open-circuit voltage; XRD: X-ray diffraction; FTIR: fourier transform infrared spectroscopy; XPS: X-ray photoelectron spectroscopy; SEM: scanning electron microscope; TEM: transmission electron microscope; P123: tergitol(tm)xh(nonionic); SDS: sodium dodecyl sulfate.

\section{Authors' contributions}

All authors (HZ, BL, QW, JS, GX, NR, ZJR, and DX) contributed jointly to all aspects of the work reported in the manuscript. $\mathrm{HZ}$ and DX performed most of the experiments and data analysis at Harbin Institute of Technology. All authors read and approved the final manuscript.

\section{Author details \\ ${ }^{1}$ School of Environment, State Key Laboratory of Urban Water Resource and Environment, Harbin Institute of Technology, 73 Huanghe Road, Nan- gang District, P.O. Box 2614, Harbin 150090, Heilongjiang, China. ${ }^{2}$ School of Electrical Engineering and Automation, Harbin Institute of Technology, Harbin 150001, China. ${ }^{3}$ The Academy of Fundamental and Interdisciplinary Science, Harbin Institute of Technology, Harbin 150080, China. ${ }^{4}$ Department of Civil, Environmental, and Architectural Engineering, University of Colorado Boulder, Boulder, CO 80309, USA.}

\section{Acknowledgements}

This study was supported by National Natural Science Foundation of China (No. 51422805, 31270004), the Science Fund for Distinguished Young Scholars of Heilongjiang Province (Grant No. JC201407), and the State Key Laboratory of Urban Water Resource and Environment (Harbin Institute of Technology (No. 2016DX10).

\section{Competing interests}

The authors declare that they have no competing interests.

\section{Funding}

National Natural Science Foundation of China (Nos. 51422805, 31470233), Science Fund for Distinguished Young Scholars of Heilongjiang Province (Grant No. JC201407), and the State Key Laboratory of Urban Water Resource and Environment (Harbin Institute of Technology (No. 2016DX10).

\section{Publisher's Note}

Springer Nature remains neutral with regard to jurisdictional claims in published maps and institutional affiliations.

Received: 5 August 2017 Accepted: 10 October 2017

Published online: 16 October 2017

\section{References}

1. Logan BE, Rabaey K. Conversion of wastes into bioelectricity and chemicals by using microbial electrochemical technologies. Science. 2012;337:686-90.

2. Liu Q, Ren ZJ, Huang C, Liu B, Ren N, Xing D. Multiple syntrophic interactions drive biohythane production from waste sludge in microbial electrolysis cells. Biotechnol Biofuels. 2016;9:162

3. Wang HM, Ren ZYJ. A comprehensive review of microbial electrochemical systems as a platform technology. Biotechnol Adv. 2013;31:1796-807.

4. Rabaey K, Verstraete W. Microbial fuel cells: novel biotechnology for energy generation. Trends Biotechnol. 2005;23:291-8.

5. Reguera G, McCarthy KD, Mehta T, Nicoll JS, Tuominen MT, Lovley DR. Extracellular electron transfer via microbial nanowires. Nature. 2005;435:1098-101.

6. Gorby YA, Yanina S, McLean JS, Rosso KM, Moyles D, Dohnalkova A, et al. Electrically conductive bacterial nanowires produced by Shewanella oneidensis strain MR-1 and other microorganisms. Proc Natl Acad Sci USA. 2006;103:11358-63.

7. Kato S, Hashimoto K, Watanabe K. Iron-oxide minerals affect extracellular electron-transfer paths of Geobacter spp. Microbes Environ. 2013;28:141-8

8. Kato S, Nakamura R, Kai F, Watanabe K, Hashimoto K. Respiratory interactions of soil bacteria with (semi)conductive iron-oxide minerals. Environ Microbiol. 2010;12:3114-23.

9. Liu F, Rotaru A-E, Shrestha PM, Malvankar NS, Nevin KP, Lovley DR. Magnetite compensates for the lack of a pilin-associated c-type cytochrome in extracellular electron exchange. Environ Microbiol. 2015;17:648-55.

10. Kato S, Hashimoto K, Watanabe K. Microbial interspecies electron transfer via electric currents through conductive minerals. Proc Natl Acad Sci USA. 2012;109:10042-6.

11. Li J, Gao H. A renewable potentiometric immunosensor based on $\mathrm{Fe}_{3} \mathrm{O}_{4}$ nanoparticles immobilized anti-lgG. Electroanal. 2008;20:881-7.

12. Zhang D, Berry JP, Zhu D, Wang Y, Chen Y, Jiang B, et al. Magnetic nanoparticle-mediated isolation of functional bacteria in a complex microbial community. ISME J. 2015;9:603-14.

13. Katz E, Lioubashevski O, Willner I. Magnetic field effects on bioelectrocatalytic reactions of surface-confined enzyme systems: enhanced performance of biofuel cells. J Am Chem Soc. 2005;127:3979-88.

14. Liu S, Yang F, Meng F, Chen H, Gong Z. Enhanced anammox consortium activity for nitrogen removal: impacts of static magnetic field. J Biotechnol. 2008;138:96-102.

15. Yavuz H, Celebi SS. Effects of magnetic field on activity of activated sludge in wastewater treatment. Enzyme Microb Technol. 2000;26:22-7.

16. Katz E, Lioubashevski O, Willner I. Magnetic field effects on cytochrome c-mediated bioelectrocatalytic transformations. J Am Chem Soc. 2004;126:11088-92

17. Li W, Sheng G, Liu X, Cai P, Sun M, Xiao X, et al. Impact of a static magnetic field on the electricity production of Shewanella-inoculated microbial fuel cells. Biosens Bioelectron. 2011;26:3987-92.

18. Yin Y, Huang G, Tong Y, Liu Y, Zhang L. Electricity production and electrochemical impedance modeling of microbial fuel cells under static magnetic field. J Power Sources. 2013;237:58-63.

19. Tao Q, Zhou S. Effect of static magnetic field on electricity production and wastewater treatment in microbial fuel cells. Appl Microbiol Biotechnol. 2014;98:9879-87. 
20. Tong Z, Yu H, Li W, Wang Y, Sun M, Liu X, et al. Application of a weak magnetic field to improve microbial fuel cell performance. Ecotoxicology. 2015:24:2175-80

21. Cheng S, Logan BE. Ammonia treatment of carbon cloth anodes to enhance power generation of microbial fuel cells. Electrochem Commun. 2007;9:492-6.

22. Wang $X$, Cheng $S$, Feng $Y$, Merrill MD, Saito T, Logan BE. Use of carbon mesh anodes and the effect of different pretreatment methods on power production in microbial fuel cells. Environ Sci Technol. 2009:43:6870-4.

23. Feng C, Ma L, Li F, Mai H, Lang X, Fan S. A polypyrrole/anthraquinone2,6-disulphonic disodium salt (PPy/AQDS)-modified anode to improve performance of microbial fuel cells. Biosens Bioelectron. 2010;25:1516-20.

24. Wang X, Falk M, Ortiz R, Matsumura H, Bobacka J, Ludwig R, et al. Mediatorless sugar/oxygen enzymatic fuel cells based on gold nanoparticlemodified electrodes. Biosens Bioelectron. 2012;31:219-25.

25. Hou J, Liu Z, Zhang P. A new method for fabrication of graphene/polyaniline nanocomplex modified microbial fuel cell anodes. J Power Sources. 2013:224:139-44

26. Tang J, Yuan Y, Liu T, Zhou S. High-capacity carbon-coated titanium dioxide core-shell nanoparticles modified three dimensional anodes for improved energy output in microbial fuel cells. J Power Sources. 2015:274:170-6.

27. Benetton XD, Navarro-Avila SG, Carrera-Figueiras C. Electrochemical evaluation of Ti/TiO2-polyaniline anodes for microbial fuel cells using hypersaline microbial consortia for synthetic-wastewater treatment. J New Mater Electrochem Syst. 2010;13:1-6.

28. Lai B, Tang X, Li H, Du Z, Liu X, Zhang Q. Power production enhancement with a polyaniline modified anode in microbial fuel cells. Biosens Bioelectron. 2011;28:373-7.

29. Li C, Zhang L, Ding L, Ren H, Cui H. Effect of conductive polymers coated anode on the performance of microbial fuel cells (MFCs) and its biodiversity analysis. Biosens Bioelectron. 2011;26:4169-76.

30. Zhang B, Du Y, Zhang P, Zhao H, Kang L, Han X, et al. Microwave absorption enhancement of $\mathrm{Fe}_{3} \mathrm{O}_{4}$ /polyaniline core/shell hybrid microspheres with controlled shell thickness. J Appl Polym Sci. 2013;130:1909-16.

31. Liang T, Wang F, Liang L, Liu M, Sun J. Magnetically separable nitrogendoped mesoporous carbon with high adsorption capacity. J Mater Sci. 2016:51:3868-79.

32. Zhu M, Diao G. Synthesis of porous $\mathrm{Fe}_{3} \mathrm{O}_{4}$ nanospheres and its application for the catalytic degradation of xylenol orange. J Phys Chem C. 2011;115:313-8.

33. Jia J, Tang Y, Liu B, Wu D, Ren N, Xing D. Electricity generation from food wastes and microbial community structure in microbial fuel cells. Bioresour Technol. 2013;144:94-9.

34. Dong H, Yu H, Wang X, Zhou Q, Feng J. A novel structure of scalable aircathode without Nafion and Pt by rolling activated carbon and PTFE as catalyst layer in microbial fuel cells. Water Res. 2012;46:5777-87.
35. Xing D, Tang Y, Mei X, Liu B. Electricity generation of microbial fuel cell with waterproof breathable membrane cathode. J Power Sources. 2015:300:491-5.

36. Lepage G, Albernaz FO, Perrier G, Merlin G. Characterization of a microbial fuel cell with reticulated carbon foam electrodes. Bioresour Technol. 2012;124:199-207.

37. Liu W, Tian K, Jiang H, Yu H. Harvest of Cu NP anchored magnetic carbon materials from Fe/Cu preloaded biomass: their pyrolysis, characterization, and catalytic activity on aqueous reduction of 4-nitrophenol. Green Chem. 2014;16:4198-205

38. Xing D, Cheng S, Regan JM, Logan BE. Change in microbial communities in acetate- and glucose-fed microbial fuel cells in the presence of light. Biosens Bioelectron. 2009;25:105-11.

39. Edgar RC. UPARSE: highly accurate OTU sequences from microbial amplicon reads. Nat Methods. 2013;10:996.

40. Wang Q, Garrity GM, Tiedje JM, Cole JR. Naive Bayesian classifier for rapid assignment of rRNA sequences into the new bacterial taxonomy. Appl Environ Microbiol. 2007:73:5261-7.

41. Hu G, Cheng M, Ma D, Bao X. Synthesis of carbon nanotube bundles with mesoporous structure by a self-assembly solvothermal route. Chem Mater. 2003;15:1470-3.

42. Li P, Xing C, Qu S, Bin LI, Shen W. Carbon dioxide capturing by nitrogendoping microporous carbon. ACS Sustain Chem Eng. 2015;3:1434-42.

43. Jia Y, Jiang J, Sun K. Pyrolysis of polyaniline-poly(styrene sulfonate) hydrogels to prepare activated carbons for the adsorption of vitamin B12. J Anal Appl Pyrol. 2015;111:247-53.

44. Bandgar DK, Khuspe GD, Pawar RC, Lee CS, Patil VB. Facile and novel route for preparation of nanostructured polyaniline (PANi) thin films. Appl Nanosci. 2014:4:27-36.

45. Sánchez-Sánchez A, Suárez-García F, Martínez-Alonso A, Tascón J. Aromatic polyamides as new precursors of nitrogen and oxygen-doped ordered mesoporous carbons. Carbon. 2014;70:119-29.

46. Chen W, Li S, Chen C, Yan L. Self-assembly and embedding of nanoparticles by in situ reduced graphene for preparation of a 3D graphene/ nanoparticle aerogel. Adv Mater. 2011:23:5679-83.

47. Malvankar NS, King GM, Lovley DR. Centimeter-long electron transport in marine sediments via conductive minerals. ISME J. 2015;9:527-31.

48. Kiseleva L, Briliute J, Khilyas IV, Simpson DJW, Fedorovich V, Cohen M, et al. Magnet-facilitated selection of electrogenic bacteria from marine sediment. Biomed Res Int. 2015. doi:10.1155/2015/582471.

49. Preston CM, Wu KY, Molinski TF, DeLong EF. A psychrophilic crenarchaeon inhabits a marine sponge: Cenarchaeum symbiosum gen. nov., sp. nov. Proc Natl Acad Sci USA. 1996;93:6241-6.

\section{Submit your next manuscript to BioMed Central and we will help you at every step:}

- We accept pre-submission inquiries

- Our selector tool helps you to find the most relevant journal

- We provide round the clock customer support

- Convenient online submission

- Thorough peer review

- Inclusion in PubMed and all major indexing services

- Maximum visibility for your research

Submit your manuscript at www.biomedcentral com/submit 